\title{
Michel Foucault: hacia una crítica política del saber económico ${ }^{1}$
}

\section{Michel Foucault: towards a political critic of the economical knowledge}

Iván Gabriel Dalmau ${ }^{2}$ Consejo Nacional de Investigaciones Científicas y Técnicas, CONICET Universidad de Buenos Aires, Argentina

Recepción: 9 de agosto del 2018

Evaluación: 13 de septiembre del 2018

Aceptación: 22 de octubre del 2018

1 Artículo resultado del pryecto UBACyT (2016-20199): L relación verdad-subjetividad en la filosofía de Michel Foucault e los años 1970 y 1980, dirigido por el profesor Dr. Marcelo Raffin.

2 Doctor en Ciencias Sociales (UBA). Becario posdoctoral del Consejo Nacional de Investigaciones Científicas y Técnicas (CONICET). Docente e investigador de la Universidad de Buenos Aires (UBA) y de la Universidad de San Martín (UNSAM).

Correo electrónico: ivandalmau@yahoo.com.ar

Dalmau, I. (2019). Michel Foucault: hacia una crítica política del saber económico. 


\title{
Resumen:
}

El objetivo del presente artículo es analizar la imbricación entre lo epistemológico y lo ontológico-político que permea a la problematización foucaulteana del saber económico realizada en el marco de su investigación genealógica respecto de la gubernamentalidad. Por lo tanto, pondremos el foco de nuestra lectura en las relaciones entre las nociones de crítica, genealogía y saber para revisitar la crítica política del saber económico realizada por Foucault. En este artículo, realizaremos entonces una lectura detenida de varios trabajos de Michel Foucault en vistas a analizar la densidad epistemoontológico-política que permea a la problematización foucaulteana del saber elaborada en su investigación genealógica respecto de la gubernamentalidad liberal y neoliberal. Cabe destacar que las formas de gobierno económico (liberalismo y neoliberalismo) no constituyen el foco de nuestro trabajo; sin embargo, analizaremos la lectura crítica desplegada por Foucault respecto del discurso de la economía política a través de sus trabajos acerca de este asunto.

Palabras clave: Foucault; Crítica; Saber; Economía política; Gubernamentalidad.

\begin{abstract}
:
The aim of this article is to analyze the overlapping between the epistemology and the ontologic-politic matter that permeates the foucaultean problematization on economical knowledge, which was done in the framework of his genealogical research about the governmentality. Thus, we will focus on this reading in the relationships between the notions of critique, genealogy, and knowledge to check again the political critiques of the economical knowledge of Foucault. In this article, we will make a deep reading in several papers of Michel Foucault in order to analyze the density epistemo-ontologic-politics that permeates the foucaultean problematization of knowledge described in his genealogical research concerning the liberal and neo-liberal governmentality. It is necessary to highlight that the ways of economical government (liberalism and neo-liberalism) do not constitute the scope of this work. However, we analyze a critical reading deployed by Foucault in his works concerning the political economy speech.
\end{abstract}

Keywords: Foucault; critique; knowledge; politicaleconomy; Governmentality 


\section{Michel Foucault: vers une critique politique de la connaissance économique}

\section{Résumé:}

L'objectif de cet article est d'analyser le chevauchement entre l'épistémologique et l'ontologico-politique qui imprègne la problématisation foucaldienne de la connaissance économique menée dans le cadre de ses recherches généalogiques sur la gouvernementalité. C'est pourquoi nous concentrerons notre lecture sur les relations entre les notions de critique, de généalogie et de connaissance pour revisiter la critique politique du savoir économique de Foucault. Dans cet article, nous ferons alors une lecture détaillée de plusieurs ouvrages de Michel Foucault en vue d'analyser la densité épistémologique-ontologiquepolitique qui imprègne la problématisation foucaldienne de la connaissance élaborée dans ses recherches généalogiques sur la gouvernementalité libérale et néolibérale. Il est à noter que les formes de gouvernance économique (libéralisme et néolibéralisme) ne sont pas au cœur de nos travaux ; nous analyserons néanmoins la lecture critique déployée par Foucault à propos du discours sur l>économie politique à travers ses travaux sur cette question.

Mots-clés: Foucault; critique; savoir; économie politique; gouvernementalité

\section{Michel Foucault: para uma crítica política do saber econômico}

\section{Resumo:}

O objetivo deste artigo é analisar a imbricação entre o epistemológico e o ontológico-político que permeia à problematização foucaulteana do saber econômico realizada no marco de sua investigação genealógica sobre a governamentalidade. Portanto, colocamos no foco de nossa leitura as relações entre as noções de crítica, genealogia e saber para revisitar a crítica política do saber econômico feita por Foucault. Neste artigo, realizaremos então, uma leitura de vários trabalhos de Michel Foucault em vista de analisar a densidade epistemo-ontológico-política que permeia à problematização foucaulteana do saber feita na sua investigação genealógica respeito à gubernamentalidad liberal e neoliberal. Também se destacam as formas de governo econômico (liberalismo e neoliberalismo) não constituem o foco de nosso trabalho; embora, analisaremos a leitura crítica desdobrada por Foucault respeito do discurso da economia política através de seus trabalhos sobre esse assunto.

Palavras-chave: Foucault; critica, saber, política econômica; governamentalidade 


\section{Presentación}

“(...) Foucault, en un procedimiento que le era propio, no ha cesado hasta el final de su vida de "releer", de resituar y de reinterpretar sus antiguos trabajos a la luz de los últimos, en una suerte de reactualización incesante” (Fontana y Bertani, 1997, p. 248).

En el presente artículo, se realiza una problematización de la imbricación entre "lo epistemológico" y "lo ontológico-político" que vertebra a la genealogía foucaultiana del saber económico. En ese sentido, al revisar el "archivo Foucault", en tanto caja de herramientas, nos detendremos en el hecho de que dichas herramientas posibilitan la realización de una crítica epistemológica que no se basta a sí misma, sino que se encuentra jalonada por objetivos ontológico-políticos ligados al diagnóstico de la actualidad. Así, en lugar de realizar un aporte al problema de la objetividad cognoscitiva de la economía política, que se articula con una concepción normativa del ejercicio de la crítica, las herramientas forjadas por Foucault permiten elaborar una crítica epistemo-ontológico-política de las formas de objetivación inmanentes a la formación de dicho saber.

Desde un punto de vista formal, dividiremos las líneas que se despliegan a continuación en dos parágrafos y un breve apartado de reflexión final. En el primero, retomando la cita que hemos colocado como epígrafe, buscaremos constituir una serie documental en torno a las nociones de crítica, genealogía y saber en el "archivo Foucault". Luego, apoyados en dicha lectura, nos enfocaremos en la crítica política del saber económico elaborada por el filósofo en el marco de su genealogía de las formas de gobierno económico.

\section{Punto de partida: crítica, genealogía y saber en el "archivo Foucault"}

"La arqueología (...) continúa jugando un rol clave en los trabajos posteriores de Foucault. Por consiguiente, la reflexión respecto del abordaje de la práctica moderna de castigo de los criminales por medio de la prisión, realizada en Vigilar y Castigar, revela que ésta involucra los cuatro aspectos fundamentales de una formación discursiva. (...) Esta aplicación de los análisis arqueológicos no es una innovación, sino más bien un retorno a las aproximaciones de Historia de la locura, en donde, por ejemplo, la arqueología mostró la estructura común del discurso filosófico cartesiano y la práctica clásica no-discursiva del encierro y reveló la relevancia moral del asilo del siglo diecinueve" (Gutting, 1989, p. 270-271).

En la primera clase del curso dictado en el Collège de France durante el ciclo lectivo de 1982-1983, es decir, en la clase del 5 de enero de 1983, Foucault se vale de una presentación del modo en que Immanuel Kant respondió a la 
pregunta "Was ist Aufklärung?", con la finalidad de inscribir su propia labor en una modulación de la crítica $^{3}$. De esta lección, consideramos pertinente remarcar la sugerente contraposición esbozada por Foucault en el siguiente fragmento:

Me parece que la elección filosófica a la que nos encontramos confrontados actualmente es ésta. Hay que optar por una filosofía crítica que se presentará como una filosofía analítica de la verdad en general, o por un pensamiento crítico que tomará la forma de una ontología de nosotros mismos, de una ontología de la actualidad. Y es esta forma de filosofía la que, de Hegel a la Escuela de Frankfurt, pasando por Nietzsche, Max Weber, etc., ha fundado una forma de reflexión a la cual, desde luego, me vinculo en la medida en que puedo (2008, p. 22).

Frente a la realización de una "analítica de la verdad en general" -preocupada por las posibilidades del conocimiento y sus límites infranqueables-Foucault practica la crítica como una ontología de la actualidad. En lugar de desplegar una gnoseología que pretenda dar cuenta de las condiciones de posibilidad del conocimiento, al normar las formas adecuadas de conocer, y una ética orientada por la búsqueda de imperativos categóricos -que permita normar las formas moralmente buenas de actuar- la crítica foucaultiana opera por medio del cuestionamiento del pretendido carácter necesario de las formas de pensamiento y de los modos de conducción de los sujetos en sus relaciones consigo mismos y con los otros (Sauquillo, 2004, pp. 167 - 185; Gros, 2008, pp. 348-361). Cabe recordar que dicha preocupación ontológico-política respecto de la actualidad se liga en la perspectiva foucaultiana a la problematización de focos de experiencia, constituidos a partir de la imbricación entre las formas de saber, las matrices normativas de comportamiento y los modos de existencia virtual para sujetos posibles (Raffin, 2015, pp. 58 - 63).

Ahora bien, retomando lo señalado en la presentación de este escrito, desplegaremos a continuación una relectura de determinados fragmentos del "archivo Foucault". La idea es revisar su problematización del saber desde la perspectiva de la inflexión del gesto crítico en que ha buscado ubicar su producción filosófica; para ello, nos apoyaremos en el característico gesto de recuperación y reelaboración recurrente que atraviesa sus trabajos. Por el momento, consideramos pertinente detenernos en la forma de

3 Pregunta kantiana en la que el filósofo se detuvo de manera recurrente entre 1978 y 1984 (Foucault, 1990: pp. 35 - 63; 1994a, pp. 562 - 578).

Dalmau, I. (2019). Michel Foucault: hacia una crítica política del saber económico. 
problematización de los discursos que Foucault propone a fines de los años sesenta en su clásico L'archéologie du savoir:

Pero de lo que aquí se trata, no es de neutralizar el discurso, de hacerlo el signo de otra cosa y de atravesar su espesor para alcanzar aquello que permanece silenciosamente más allá de él sino, al contrario, de mantenerlo en su consistencia, de hacerlo surgir en la complejidad que le es propia (...). Sustituir el tesoro enigmático de las "cosas" anteriores al discurso, por la formación regular de los objetos que no se perfilan más que en él. Definir esos objetos sin referencia al fondo de las cosas, sino en relación al conjunto de las reglas que permiten formarlos como objetos de un discurso y constituyen así sus condiciones de aparición histórica (Foucault, 1969, p. 65).

En dicho contexto, remarcará Foucault que la problematización arqueológica de los discursos consiste en abordarlos como prácticas y en dar cuenta de sus condiciones de posibilidad. Con esto se logra el establecimiento de los modos históricos de constitución de ciertas positividades, en lugar de tomarlas de antemano como evidencia y punto de partida. Así lo señala Maria Muhle:

La puesta en cuestión del documento realizada por la arqueología foucaultiana se dirige, por lo tanto, en contra de un modelo de historia que aspira al reconocimiento de un pasado inscripto en los documentos y luego desvanecido. El documento no es más para la historia el rastro de las cosas pasadas, "la materia inerte de la historia", por el contrario, es en el "tejido documentario" mismo que la historia busca definir las unidades, los conjuntos, las series, las relaciones. El documento, en su materialidad, es el objeto de la historia-sin, por lo tanto, reenviar a la existencia de las cosas, de los objetos naturales o los estados, sabiendo que, en palabras de Paul Veyne, esas entidades no son más que correlatos de las prácticas discursivas (2012, p. 190).

En función de lo anterior, Foucault propondrá la noción de formación discursiva, la cual caracteriza del siguiente modo:

En el caso en que se pudiera describir, entre un cierto número de enunciados, un semejante sistema de dispersión, en el caso en que entre los objetos, los tipos de enunciación, los conceptos, las elecciones temáticas, se pudiera definir una regularidad (un orden, correlaciones, posiciones y funcionamientos, transformaciones), se dirá, por convención, que se trata de una formación discursiva, -evitando así palabras demasiado cargadas de condiciones y de consecuencias, inadecuadas por otra parte para designar una dispersión semejante, como "ciencia", o "ideología", o "teoría", o "dominio de objetividad"”. Se llamarán reglas de formación a las condiciones a las que están sometidos los elementos de esta repartición (objetos, modalidad de 
enunciación, conceptos, elecciones temáticas). Las reglas de formación son condiciones de existencia (pero también de coexistencia, de conservación, de modificación y de desaparición) en una repartición discursiva determinada (Foucault, 1969, p. 53).

El pasaje de la problematización de los objetos y las modalidades enunciativas hacia sus reglas de formación permite desanclar la reflexión epistemológica del interior de la relación sujeto-objeto y visibilizar los términos de la relación cognoscitiva como inmanentes a dichas reglas de formación. La arqueología no pretende configurarse como una teoría del conocimiento alternativa, que según la interpretación foucaultiana problematizaría la relación sujetoobjeto, sino que entre sus objetivos se destaca el dar cuenta de los modos históricos de constitución de ambos términos al remitirlos a sus condiciones de posibilidad. De este modo, en lugar de problematizar las posibilidades del conocimiento y sus límites infranqueables, configura un registro epistemológico que no se "basta a sí mismo", sino que se encuentra jalonado por preocupaciones ontológico-políticas. De lo que se trata, entonces, es de dar cuenta de la formación inmanente de los objetos y las posiciones de los sujetos, a partir del abordaje del discurso de las ciencias respecto de la vida, el trabajo, el lenguaje y las ciencias humanas.

A medida que la arqueología se despliega a través del abordaje de la historia efectiva de las prácticas discursivas, también va ubicándose en una posición de exterioridad con respecto a los discursos de cuya formación se ocupa. En ese sentido, la arqueología permite llevar a cabo una indagación que no se encuentra motivada por preocupaciones normativas; además, su trabajo de archivo evita tomar a la ciencia natural como patrón para la realización de una historia en clave teleológica. De ello se puede dar fe en el prefacio de su trabajo precedente, Les mots et les choses, de 1966:

No se interrogará a los conocimientos descritos en su progreso hacia una objetividad en la que la ciencia actual pudiera reconocerse; lo que se pondrá en juego, es el campo epistemológico, la episteme en que los conocimientos, vistos más allá de todo criterio referente a su valor racional o sus formas objetivas, hunden su positividad y manifiestan así una historia que no es la de su perfección creciente, sino más bien la de sus condiciones de posibilidad (...). Más que de una historia en el sentido tradicional del término, se trata de una "arqueología" (Foucault, 1966, p. 13).

Como se dijo, la problematización del saber se encuentra desvinculada del interior de la relación sujeto-objeto, prescinde de una concepción teleológica 
de la historia basada en las ciencias naturales y evita llevar a cabo una reflexión epistemológica de carácter normativo (Castro, 1995, p. 39). De este modo, en lugar de "bastarse a sí mismo", el trabajo epistemológico del arqueólogo se encuentra motivado por objetivos ontológico-políticos. Lo anterior quiere decir que la arqueología del saber habilita a una forma de problematización de los saberes que contribuye a la realización de un diagnóstico de la actualidad (a una ontología de nosotros mismos), en la misma medida en que permite dar cuenta del modo en que históricamente $-\mathrm{y}$ de manera inmanente a cierta disposición epistémica- se constituyen objetos y posiciones de sujeto. En suma, en lugar de contribuir a la crítica normativa respecto de la objetividad cognoscitiva de las ciencias empíricas y las ciencias humanas, la crítica arqueológica se desplaza hacia la problematización de las formas de objetivación.

Dejando a un lado la discusión de los vínculos de Foucault con el estructuralismo, cabe recordar que, en una entrevista que le hicieran en 1967, sostuvo que el estructuralismo "permite diagnosticar qué es el hoy" (Foucault, 1994b, pp. 580-584). En la misma línea, cabe destacar que en un entrevista realizada en el contexto de la publicación de Les mots et les choses (Foucault, 1966), el filósofo planteó que:

(...) A propósito de Nietzsche, podemos volver sobre su pregunta: para él, el filósofo es quien diagnostica el estado del pensamiento. Por cierto, se pueden concebir dos clases de filósofos, el que abre nuevos caminos al pensamiento, como Heidegger, y el que juega en cierta forma el rol de arqueólogo, que estudia el espacio en el que se despliega el pensamiento, así como sus condiciones, su modo de constitución (Foucault, 1994c, p. 553).

Ahora bien, dado que la crítica genealógica del saber económico constituye el foco de nuestro trabajo, antes de dar paso al siguiente apartado, se nos impone la tarea de explicitar lo que podría denominarse como "ecos arqueológicos de la genealogía". En línea con la lectura condesada en la cita de Gutting que hemos colocado como epígrafe del presente apartado, por "ecos arqueológicos de la genealogía" nos referimos tanto la centralidad que posee la noción de saber dentro de las herramientas de que Foucault se vale en sus genealogías, como así también al modo recurrente en que se ocupa del discurso de las ciencias de la vida, el trabajo, el lenguaje y las ciencias humanas. Además, no puede soslayarse la problematización foucaultiana de la genealogía como un método que permite realizar un trabajo filosófico en las canteras de la historia que no se encuentra jalonado por una preocupación normativa, ni adopta una perspectiva historiográfica de carácter teleológico (Villacañas y Castro, 2018, pp. 12-22). 
Al respecto, cabría recordar que en el marco de la Leçon sur Nietzsche, dictada en Montreal en 1971, el filósofo se enfocó en la posibilidad erigida, a partir de la genealogía nietzscheana, de: “(...) pensar el conocimiento como un proceso histórico previo a toda problemática de la verdad, y más fundamentalmente que en la relación sujeto-objeto. El conocimiento liberado de la relación sujeto-objeto, es el saber" (Foucault, 2011, p. 205). En el resumen del primero de sus cursos dictados en el Collège de France, nos referimos a Leçons sur la volonté de savoir, correspondiente al ciclo lectivo 1970-1971, Foucault explicitaría el eco arqueológico que permeaba a la genealogía que se encontraba desarrollando:

Investigaciones realizadas previamente permitieron reconocer un nivel singular entre aquellos que permiten analizar a los sistemas de pensamiento: el de las prácticas discursivas. Se trata de una sistematicidad que no es de tipo lógico ni lingüístico. Las prácticas discursivas se caracterizan por el recorte de un campo de objetos, la definición de una perspectiva legítima para el sujeto de conocimiento, la fijación de normas para la elaboración de conceptos y teorías. Cada una de ellas supone, entonces, un juego de prescripciones que rigen las exclusiones y elecciones. (...) Los caracteres generales de esas prácticas y los métodos propios para analizarlas se inventariaron bajo el nombre de arqueología (2011, pp. 217-218).

En 1971, Michel Foucault publicará además su clásico artículo titulado "Nietzsche, la généalogie, l'histoire", el cual constituye una referencia ineludible para los que pretendemos dar cuenta del "eco arqueológico" que atraviesa a la reflexión genealógica. En dicho artículo, Foucault retoma la distinción nietzscheana entre Ursprung y Erfindung, términos alemanes que implican la noción de "origen" e "invención", respectivamente. Por lo tanto, en la lectura foucaultiana de Nietzsche, Ursprung es vinculado con la noción metafísica de "origen fuente", de "origen transhistórico", mientras que Erfindung se liga a la problemática concreta de la procedencia (Herkunft) y de las condiciones de posibilidad para la emergencia o surgimiento (Entstehung) de las prácticas. En términos del propio Foucault: "la procedencia permite rencontrar bajo el aspecto único de un carácter, o de un concepto, la proliferación de los acontecimientos a través de los cuales (gracias a los cuales, contra los cuales), ellos se formaron" (1994d, p. 141).

En la lectura de Nietzsche propuesta por Foucault, el análisis de la emergencia, es decir, la indagación respecto de las denominadas condiciones de su posibilidad, constituye una herramienta que viabiliza la introducción 
de las fuerzas y sus correlaciones en el marco del abordaje genealógico de los problemas filosóficos. Forma de problematización del método de trabajo filosófico que será retomada de manera recurrente por el filósofo a lo largo de la década del setenta, por ejemplo en el marco de sus estudios sobre las prisiones. Al respecto, el especialista Marcelo Raffin sostuvo que:

Foucault se propone mostrar cómo las prácticas sociales pueden llegar a engendrar dominios de saber que no sólo hacen que aparezcan nuevos objetos, conceptos y técnicas, sino que hacen nacer, además, formas totalmente nuevas de sujetos y sujetos de conocimiento. En este sentido, Foucault afirma que el sujeto de conocimiento posee una historia, la relación del sujeto con el objeto, y, más claramente, la verdad misma tiene una historia (2014, p. 130).

A finales de la década del setenta, justamente en el marco de la genealogía del saber económico en la que nos detendremos en el próximo apartado, Foucault propuso llevar a cabo una crítica política del saber. Crítica a la caracterizó del siguiente modo:

La crítica que les propongo consiste en determinar bajo qué condiciones y con qué efectos se ejerce una veridicción, es decir, una vez más, un tipo de formulación dependiente de ciertas reglas de verificación y falseamiento. (...) No es la historia de lo verdadero, no es la historia de lo falso, es la historia de la veridicción la que posee importancia política (2004a, pp. 37 -38).

De lo que se trata, entonces, es de indagar respecto de las condiciones que hicieron posible que se produjese una articulación entre una serie de prácticas y un régimen de veridicción, cuyos efectos serían que algo que no existía, siguiese sin existir pero, sin embargo, se inscribiese en "lo real". En ese sentido, frente a la puesta en cuestión del discurso científico decimonónico en términos de "conocimiento superado" por la ciencia actual, cuyo carácter político se ligaría con el hecho de que se trataba de "conocimiento todavía no suficientemente elaborado" que tendría por presunta función la "legitimación del poder", la crítica política del saber llevada a cabo por el genealogista permite dar cuenta del modo en que en determinado momento histórico se produjo un acoplamiento entre una serie de prácticas y un régimen de veridicción.

Es decir, que tal como se desprende de la palabra foucaultiana, la problematización de los saberes apuntará a dar cuenta del modo en que los susodichos articularon una serie de prácticas, al constituir ciertos objetos pasibles de ser interrogados a partir de determinadas reglas de verificación 
y falseamiento. Por lo tanto, en lugar de llevar a cabo una crítica normativa respecto de la objetividad cognoscitiva de dichos saberes, la crítica genealógica problematiza las formas de objetivación.

Antes de dar paso al siguiente apartado, consideramos pertinente recapitular la estrategia de lectura elaborada, que se sostiene básicamente a partir de la puesta en juego de tres pares categoriales, dos introducidos por el propio Foucault y el tercero incorporado por quien escribe estas líneas. La articulación de los pares introducidos por Foucault permitió perfilar dos senderos para el trabajo filosófico que, declinado hacia el abordaje del saber económico, daría lugar a un tercer par. Tenemos, entonces, una filosofía analítica de la verdad en general, preocupada por las posibilidades del conocimiento y sus límites infranqueables, es decir, por la elaboración de una teoría del conocimiento que, respecto de la economía política, permitiría perfilar una crítica epistemológica normativa acerca de su objetividad cognoscitiva. Frente a lo cual, la propuesta foucaultiana de llevar a cabo una ontología de la actualidad se entronca con el abordaje arqueológico del saber y da lugar a una crítica epistemológica que -en lugar de bastarse a sí misma- se configura como un aporte a la problematización ontológico-política de la actualidad, por medio de la crítica de las formas de objetivación inmanente a la constitución del saber económico.

\title{
Gubernamentalidad y objetivación: reflexiones en torno a la crítica genealógica del saber económico
}

\begin{abstract}
“(...) Luego de la descripción de los dispositivos de seguridad y de los nuevos problemas planteados por la gestión de la población urbana, la exposición se desplaza hacia lo que finalmente constituye el marco general en el que hay que situar todas sus investigaciones sobre el poder: la historia de la gubernamentalidad" (Castro, 2011, p. 59).
\end{abstract}

Los cursos dictados por el filósofo en el Collège de France durante la segunda mitad de la década del setenta, editados en formato libro entre 1997 y 2004 (Foucault, 1997, 2004a, 2004b), constituyen lo que la crítica ha denominado "cursos biopolíticos". En el primero de ellos, dictado en 1976 (Foucault, 1997), la biopolítica es abordada en la última lección del curso, en el marco de la problematización de las mutaciones del discurso de la guerra de razas. Si bien no pueden desconocerse los matices, ni las diferencias de acento, cabría destacar que el modo en que problematiza allí el "poder sobre la vida", que se distingue del "poder de espada del soberano", es bastante cercano al elaborado en el capítulo final del primer tomo de Histoire de la sexualité (Foucault, 
1976), publicado el mismo año en que dictara el curso. En los cursos siguientes, tomando como punto de partida el análisis de los dispositivos de seguridad (Foucault, 2004b), el foco de problematización se desplaza desde la biopolítica hacia su marco de racionalidad: el liberalismo (Foucault, 2004a). Al respecto, el investigador Davide D'Alessandro planteó que:

Foucault orientó su curso del año 1978, Seguridad, Territorio, Población, hacia un horizonte amplio que implica a la biopolítca: una historia de la gubernamentalidad. (...) En el pensamiento de Foucault, el objetivo es comprender la ligazón entre los regímenes de verdad y las prácticas de gobierno político y económico que gestionan la vida. La biopolítica, por medio de los "discursos de veridicción" (de la biología y de la economía), objetiva al hombre, como ser biológico-viviente y como actor productivo/ consumidor (2011, p. 57).

No puede pasarse por alto que si en el curso del año 1976 Foucault caracterizaba a los dispositivos biopolíticos, que tienen por blanco la regulación de la vida biológica de la población, como modos de "estatización de lo biológico" (Foucault, 1997, pp.213-235), en los cursos consecutivos, articulados en torno a la problematización de las formas modernas de gubernamentalidad, propondrá elaborar un análisis que prescinda de tomar como punto de partida "al objeto, la institución y la función" (Foucault, 2004b, pp.91 - 118) y, por el contrario, inscribirá su indagación en el marco de la apuesta teórica y metodológica nominalista que consiste en "suponer que los universales no existen" (Foucault, 2004a, pp. 3 - 28). De allí que, en lugar de problematizar el Estado como un "monstruo frío" cuya historia pudiera trazarse por sí misma, o de convertirlo en un mero reflejo de las transformaciones en las relaciones de producción, propondrá analizar su configuración y sus mutaciones como pliegues y peripecias en la historia de las prácticas gubernamentales (Lazzarato, 2005, p. 1).

De lo que se trata, entonces, es de llevar a cabo una indagación que prescinda de tomar como punto de partida el sujeto, el Estado y el mercado en tanto términos universales. Apuesta que Foucault caracterizará como "antihistoricista", en la medida en que, en lugar de "pasar los universales por el rallador de la historia", consiste más bien en ponerlos en cuestión y ver qué historia puede hacerse. Al caracterizar sus indagaciones respecto de las formas modernas de gubernamentalidad, sostuvo Foucault que:

He querido estudiar el arte de gobernar, es decir la manera reflexionada de gobernar mejor y además, al mismo tiempo, la reflexión sobre la mejor manera posible de gobernar. Es decir que intenté abordar la instancia de la reflexión 
dentro de la práctica de gobierno y acerca de la práctica de gobierno. (...) Intenté determinar la manera a través de la cual se ha establecido el dominio de la práctica del gobierno, sus diferentes objetos, sus reglas generales, sus objetivos de conjunto, con el fin de gobernar de la mejor manera posible. En suma, es (...) el estudio de la racionalización de la práctica gubernamental dentro del ejercicio de la soberanía política (2004a, p. 4).

En la problematización foucaultiana desarrollada a lo largo de los cursos dictados en el Collège de France durante los ciclos lectivos de 1977-78, Sécurité, Territoire, Population (Foucault, 2004b), y 1978-79, Naissance de la biopolitique (Foucault, 2004a), el problema de la emergencia de la biopolítica y los dispositivos de seguridad es situado dentro del marco de la realización de una "historia de la gubernamentalidad", y específicamente en el seno de lo que Foucault llamará, en la última lección del curso de 197778, "gubernamentalidad de los economistas" (2004b, pp. 341 - 370), lo cual será retomado en el curso del año siguiente, en el que propondrá: "estudiar el liberalismo como marco general de la biopolítica" (2004a, p. 24).

Desde las primeras lecciones del curso de 1977-78, se advierte la centralidad que para Foucault posee la formación de la economía política en el surgimiento de las formas modernas de gubernamentalidad y en la constitución del objeto población. De hecho, cuando al final del curso contraponga la "gubernamentalidad de los economistas" a la de "los políticos", propia del arte de gobierno según el principio de la Razón de Estado, parte de la estrategia argumentativa consistirá en distinguir y analizar el modo en que fisiócratas y mercantilistas ponen en consideración a la población en el seno de sus problematizaciones.

Es decir, que Foucault se detendrá en el hecho de que la población deja de ser un mero dato cuantitativo más, en el seno del análisis "mecánico" respecto de la "fuerza relativa" de los diferentes Estados dentro del marco de la denominada "balanza Europea", a cobrar un espesor y una densidad que la tornan un objeto de problematización privilegiado. De "mero dato" para el análisis de las riquezas, devendrá en una "realidad espesa" atravesada por dinámicas que, paradójicamente, escapan a una matriz mecánica de problematización, y que se convierte en blanco privilegiado del gobierno económico, tal como fuera problematizado por la naciente economía política de la mano de François Quesnay y la Escuela Fisiocrática francesa. Gobierno económico de la población que se erigirá en torno a la clásica fórmula laissez faire-laissez passer y que se articulará estratégicamente a través de dispositivos de seguridad (Foucault, 2004b, pp. 91 - 118). 
Al respecto, consideramos elocuente que, dentro del marco del denominado "análisis de las riquezas", Foucault remarque que la población poseía un estatuto ambiguo, al punto de llegar a sostener que ésta se encontraba "presente y ausente" dentro de la grilla forjada por dicho saber. El análisis de las riquezas objetiva a la población como una variable más para dar cuenta de la fuerza relativa de los Estados, mientras que la mutación introducida por la economía política consistirá en que dicha población será objetivada como "realidad densa" frente al ejercicio del gobierno. De este modo, la mencionada "presencia-ausencia" alude a la discontinuidad entre ambas formas de objetivación.

Foucault problematiza la mencionada discontinuidad epistémica en el marco de su abordaje genealógico de las transformaciones en las formas de problematización del ejercicio del gobierno. Desde dicha perspectiva, destaca la imbricación entre la constitución del objeto población y la emergencia del liberalismo como racionalidad de gobierno, es decir, como forma reflexiva de gobernar erigida en torno a la formación de la economía política y articulada tecnológicamente por medio de dispositivos de seguridad:

Así, la misma naturalidad que torna refractaria a la población con respecto a los decretos del soberano, la vuelve, al mismo tiempo, accesible a técnicas de transformación, bajo la condición de que ellas sean esclarecidas, reflexionadas y calculadas: para actuar sobre ella deberán, en efecto, hacer jugar una serie de factores que se encuentran aparentemente alejados de la población (por ejemplo, los impuestos, la infraestructura, los flujos monetarios), pero a los cuales se encuentra ligados. (...) La población no es, entonces, ni una colección de sujetos jurídicos relacionados con una voluntad soberana, ni un conjunto de cuerpos individuales atrapados por instituciones disciplinarias. Ella reenvía, más bien, a una masa que se inserta dentro del régimen general de los seres vivientes y ofrece, por el mismo motivo, una superficie de agarre para transformaciones reflexivas y calculadas (Mauer, 2015, p. 46).

A mayor abundancia con respecto a la centralidad de la población en tanto objeto de saber y blanco de intervención política, perfilada tácticamente a través de los dispositivos de seguridad que caracterizan a las tecnologías de gobierno predominantes dentro de las formas de gobierno económico, nos remitimos a la lectura realizada por Sabot (2016) respecto de la problematización foucaultiana de las distintas formas de normalización que se configuran en la modernidad. Lectura que nos permite, además, enfatizar que el desplazamiento desde la biopolítica hacia la historia de la 
gubernamentalidad implicó un refinamiento conceptual y un replanteamiento del foco de miras, más no un abandono de la problemática del biopoder, con sus polos disciplinario y biopolítico:

En efecto, a finales de los años setenta Foucault realiza un desplazamiento con respecto a su análisis que pasa a través de una distinción interesante entre dos modos de normalización. (...) Foucault completa el análisis de la operación normación-conformación por referencia a la dimensión de otras "técnicas de normalización" que reenvían, en realidad, a otra lógica normativa, inmanente y relativa a los denominados "dispositivos de seguridad". Esta lógica no parte de una norma preestablecida, fijada de antemano a la actividad de corrección y orientación como un modelo o ideal a seguir. Cuando examina las llamadas sociedades de seguridad, Foucault muestra, en efecto, que las operaciones de normalización no se apoyan sobre una norma exterior, sino que toman apoyo sobre las diferencias entre las normalidades y las hacen jugar unas contra otras (Sabot, 2016, p. 5).

En este contexto, se destaca el carácter indisociable del surgimiento del liberalismo como matriz de problematización del ejercicio del gobierno y la constitución de la economía política; saber cuya formación se encuentra estrechamente ligada a la emergencia de la problematización del mercado como ámbito de veridicción (Foucault, 2004a, pp.29 - 51). Podría decirse, entonces, que nos encontramos frente a una doble mutación: así como la población deja de ser un "mero dato", y deviene en "realidad densa" frente al ejercicio del poder, el mercado pasa de ser un mero ámbito de jurisdicción, blanco de políticas de controles de precios, por ejemplo, a constituirse en ámbito de producción de la verdad.

De este modo, al aparecer "con espesor propio" frente al gobierno, el respeto a los "mecanismos del mercado" emergerá como una limitación interna al ejercicio del gobierno, de ahí la "célebre" consigna fisiocrática que hemos mencionado previamente. De hecho, ya no se trata de oponer una limitación externa al ejercicio del gobierno, mediante una apelación a cuestiones jurídicas -la violación de un derecho, por ejemplo -, sino que las "verdades del mercado" operan como un filtro intrínseco a la práctica del gobierno, que de no "respetarlas" no comete una injusticia sino una torpeza cuyos efectos serán irremediablemente contrarios a lo buscado. En términos de la investigadora Johanna Oksala:

Foucault sostiene que con el desarrollo de la economía política se estableció un nuevo principio para la limitación de la racionalidad gubernamental. Mientras que hasta ese momento la ley había funcionado como una limitación externa 
al gobierno excesivo, el nuevo principio -economía política-era interno a la misma racionalidad gubernamental. Esto significa que el gobierno no tenía que limitarse a sí mismo porque violara la libertad o los derechos básicos de los hombres, sino en vistas del aseguramiento de su propio éxito. (...) En su momento, esto hizo posible juzgarlas como buenas o malas [a las prácticas gubernamentales], no en los términos de algún principio legal o moral, sino en términos de verdad: proposiciones sujetas a la división entre lo verdadero y lo falso. De acuerdo con Foucault, la actividad gubernamental entró, entonces, en un nuevo régimen de verdad (2013, p. 57).

Las prácticas gubernamentales serán susceptibles de ser analizadas no en términos de justicia e injusticia, sino de adecuación e inadecuación a las verdades inmanentes al mercado, cuyo respeto resulta fundamental para el "éxito" del gobierno. Así, se consolida lo que Foucault denomina como un "gobierno frugal", una suerte de "naturalismo" que hace del mercado una zona vedada para la acción gubernamental; ya que si el gobierno pretende ser exitoso, no puede desconocer ni intentar "espuriamente" violentar sus mecanismos (Foucault, 2004a). Sostenemos, entonces, que en el marco de la genealogía del liberalismo, en tanto prisma reflexivo gubernamental, el filósofo elabora una crítica epistemológica y ontológico-política respecto de las formas de objetivación inmanentes a la formación del discurso de la economía política. Lo que significa que no se trata de desplegar una crítica normativa que denuncie la "falta de objetividad cognoscitiva" de la ciencia económica, sino de problematizar el discurso de la economía política en vistas a desbrozar qué objetos se constituyeron de modo inmanente a la formación de dicho saber, al inscribirse estratégicamente en "lo real".

En ese sentido, el liberalismo se consolida -en estrecha vinculación con la formación del discurso de la economía política-como una forma de "gobierno esclarecido", cuyo ejercicio no puede desconocer la "realidad espesa" del mercado, a cuyas verdades debe adecuarse. Como lo planteara Nosetto (2010), en la lectura foucaultiana, el liberalismo configura una racionalidad de gobierno que:

[p]ostula la evidencia de los procesos naturales de intercambio. Es un discurso atento a la naturaleza de las relaciones entre hombres y cosas que deduce, a partir de ella, la conveniencia, la utilidad y las limitaciones del ejercicio del gobierno. El mercado se constituye en el lugar de manifestación de la naturalidad de estos procesos, espacio de aparición, de evidencia de la mecánica natural de los intercambios. El mercado es postulado, de esta manera, como el lugar de veridicción, como el espacio de manifestación de 
los mecanismos naturales, donde se revela una verdad que se constituye en parámetro de verificación y falseamiento de toda práctica gubernamental (2010, p. 210).

De lo que se trata es de la conformación de una forma esclarecida de gobernar, cuya matriz estratégica de reflexión se perfila a través de la grilla de inteligibilidad inmanente al discurso de la economía política. Encontramos allí una crítica de la economía política en tanto saber que, por ende, se coloca por fuera de la relación sujeto-objeto y prescinde de una preocupación epistemológica normativa; por el contrario, el trabajo epistemológico de archivo sobre la formación del discurso económico se encuentra jalonado por objetivos ontológico-políticos, puesto que apunta a dar cuenta de la manera en que las formas de objetivación inmanentes a dicho saber configuran la grilla a partir de la que se definen los blancos de la práctica gubernamental y los criterios para evaluarlas, con lo que se forma, por ejemplo, "la población" y "el mercado" como objetos de un discurso cuya modalidad enunciativa configura al economista como sujeto legítimo de enunciación de la verdad acerca de cómo gobernar.

La historia de las formas modernas de gubernamentalidad desplegada en estos cursos es completada por Foucault por medio del abordaje genealógico de las inflexiones contemporáneas de la racionalidad política liberal, razón por la cual le dedicará gran parte del curso correspondiente al ciclo lectivo de 1978-79 al tema del neoliberalismo (Foucault, 2004a), fundamentalmente en sus formas alemana y norteamericana. En dicho contexto, sostuvo Foucault que el neoliberalismo, formado en la Alemania de entreguerras y consolidado en la posguerra, se encuentra ligado fundacionalmente a la Escuela de Friburgo, a la publicación de la revista Ordo y a un conjunto de economistas, sociólogos y juristas, entre quienes se destacan Ludwig von Mises, Friedrich von Hayek, Walter Eucken y Wilhelm Röpke, entre otros (Botticelli, 2014, pp. 133 - 135; Méndez, 2017, pp. 112 - 146). Allí, remarcará el filósofo que el ordoliberalismo -denominación que se le otorga a esta corriente en alusión al título de la citada publicación- se articuló tomando el nazismo como campo de adversidad, al problematizarlo como "punto de coalescencia" en el que convergen las distintas formas de dirigismo y planificación económica, como también las políticas sociales de corte "socialista" (en un sentido amplísimo, lo que implica una radical puesta en cuestión de las políticas de redistribución progresiva del ingreso).

En este contexto, hacemos propia la reconstrucción de la lectura foucaultiana propuesta por el investigador Mauro Benente: 
El neoliberalismo alemán no es idéntico al norteamericano, pero ambos tienen como enemigos comunes al keynesianismo y a la dirección estatal de la economía, y al igual que el liberalismo, el neoliberalismo es una racionalidad de gobierno (...). La legitimación del Estado a partir de la economía, fue posible a partir de una relectura del nazismo y del funcionamiento del Estado, ya que el ordoliberalismo postuló que éste, desde el punto de vista económico, no había sido una monstruosidad ni una incoherencia económica, y en definitiva el New Deal, el plan Beveridge, la planificación soviética y el nazismo pusieron en juego los mismos principios (2015, p. 285-286).

En el marco del curso, Foucault remarcará la mutación que había introducido el neoliberalismo respecto del liberalismo clásico, al destacar lo inapropiado de una crítica que "denuncie" que los neoliberales pretenden volver al siglo XIX. Básicamente, sostuvo que con la Escuela de Friburgo se rompe la ligazón entre liberalismo y laissez-faire, el "naturalismo" al que hemos referido previamente, ya que ellos señalarán que el mercado no es "algo dado", una suerte de "dato natural", sino que debe ser constituido activamente. Es decir, que la mutación de la racionalidad gubernamental se encuentra profundamente imbricada con la ruptura en la forma en que en el discurso de la economía política se constituye el objeto "mercado".

Dicho de otro modo, la transformación en la racionalidad gubernamental se encuentra imbricada con la mutación de las formas de objetivación inmanentes a la formación del discurso económico. Así, más que gobernar limitando la acción del gobierno en función del "respeto" a los mecanismos del mercado, propondrán que hay que gobernar activamente para producir las condiciones del mercado. Por lo tanto, nos encontramos con la problematización del mercado como ámbito que debe ser configurado formalmente a partir de técnicas gubernamentales que promuevan la empresarialización de las relaciones sociales.

De lo que se trata, entonces, es de un activo gobierno del marco que permita inscribir en "la realidad" el mecanismo de la competencia; si bien cualquier intervención sobre los mecanismos del mercado será impugnada, se alentarán formas de intervención activas sobre las condiciones de posibilidad del mercado. Más que de un retorno al "naturalismo" del siglo XIX, se trata de un "liberalismo sociológico" que, en lugar de tomar el mercado como dato y límite, lo problematiza bajo la forma de la competencia en tanto principio formal que debe ser inscripto en "lo real" (Foucault, 2004a, pp. 135 - 164). En ese sentido, Castro-Gómez (2010) sostiene que desde la perspectiva genealógica foucaultiana: 
[e]1 ordoliberalismo alemán no es un "regreso" al liberalismo clásico del siglo XVIII, sino una tecnología completamente diferente. Foucault muestra que la principal diferencia con el liberalismo clásico es su concepción antinaturalista del mercado. La gubernamentalidad liberal se basaba en el laissez-faire, pues suponía que el mercado tiene sus "leyes internas", de carácter natural, sobre las que no hay que intervenir. Los ordoliberales alemanes plantean, en cambio, una economía de mercado sin laissez-faire. El mercado, para ellos, no es una "realidad natural", sino que requiere de la intervención política. Tampoco la competencia de intereses es un "dato natural" (como decían los empiristas ingleses), sino que debe ser consecuencia de una intervención. El problema para los ordoliberales ya no es intervenir o no intervenir (agenda o non agenda), como para el liberalismo clásico, sino saber cómo intervenir (2010, p. 183).

La torsión producida por el ordoliberalismo sería profundizada y radicalizada en el marco del desarrollo de la "teoría del capital humano" por parte de la Escuela de Chicago, es decir del neoliberalismo norteamericano, entre cuyos exponentes se destacan Friedrich von Hayek, Milton Friedman y Gary Becker. La teoría del capital humano se erige a partir de la problematización del capital como "aquello que produce un beneficio", en el contexto de "asignación de recursos limitados hacia fines mutuamente excluyentes", lo que permite la introducción de un desbloqueo epistemológico al posibilitar la inclusión del trabajo como actividad dentro del análisis económico (Foucault, 2004a, pp. 221 - 244). El "capital humano", en tanto objeto, se constituirá entonces en torno a una serie de capacidades físicas e intelectuales vinculadas a la "productividad" y al savoir-faire atravesadas por la tensión entre lo "innato y lo adquirido". En términos del politólogo Emiliano Sacchi:

Si el ordoliberalismo había comenzado extendiendo la racionalidad económica empresarial hacia todo el campo social, los neoliberales norteamericanos van a radicalizar este principio, hasta anular la distinción entre lo económico, lo social y el actuar humano. No se trata ya de extender los mecanismos económicos a la sociedad, sino de hacer de lo social una economía y, sobre todo, de toda actividad humana un comportamiento que asigna recursos escasos a fines antagónicos (Sacchi, 2017, p. 50).

En el seno de dicha estrategia discursiva, la "grilla de análisis económico" es aplicada a la totalidad de las prácticas sociales, incluso a aquellos comportamientos considerados "habitualmente" como "no económicos" (educación, relaciones familiares, dieta y acceso a la salud) serán problematizados en términos de "inversiones en capital humano". Es decir, 
una vez más, los blancos y criterios para racionalizar el ejercicio del gobierno se modifican en estrecha ligazón con la transformación en las formas de objetivación inmanentes al discurso económico. Justamente, en la medida en que la constitución del capital humano en tanto objeto habilita la aplicación de la grilla económica como forma de inteligibilidad de todas las prácticas sociales y se perfila como criterio de intervención gubernamental. Por lo tanto, la contracara de que la racionalidad económica sea problematizada como el modo adecuado y sistemático de responder a las transformaciones de las variables del medio, es que el blanco del ejercicio del gobierno se tornará eminentemente gobernable, a través de las intervenciones "esclarecidas" sobre el juego entre dichas variables (Foucault, 2004a, pp. 245 - 270; Cadahia y Blengino, 2018, pp. 430-433).

\section{Palabras finales}

"La arqueología puesta al servicio de un saber crítico respecto de la experiencia, de las formas de pensar, de los comportamientos del presente, no los presenta como universales, como objetos existentes desde siempre, "ni encarnaciones de una esencia, ni individualización de una especie”, sino más bien como una (...) positividad; la genealogía se interroga acerca de cuáles condiciones, discursos y prácticas los han puesto en marcha y cuál conexión de saber-poder los ha vuelto aceptables, tornando evidente lo que de por sí no lo era" (Giordano, 2007, p. 66).

Hemos revisado la crítica genealógica elaborada por Michel Foucault respecto del discurso de la economía política, para lo cual nos hemos ocupado previamente de trabajar con el "archivo Foucault" en vistas a constituir una serie, una trama, entre las nociones de crítica, genealogía y saber. Luego, colocamos el foco de lectura en la problematización de la economía política elaborada por el filósofo en el marco de su abordaje de las formas de gobierno económico, es decir, del liberalismo y el neoliberalismo en sus vertientes alemana y norteamericana. En ese sentido, consideramos pertinente destacar que el propio Foucault sostuvo, en el contexto del curso Du gouvernement des vivants (Foucault, 2012, pp. 71 - 89) -curso inmediatamente posterior a Naissance de la biopolitique - que el objetivo de sus trabajos consistía, en parte, en dar cuenta de la imbricación que liga a lo que suele denominarse como "lo epistemológico" y "lo político".

Esta imbricación nos permite captar la potencia crítica que atraviesa a las herramientas forjadas por Foucault; nos referimos, específicamente, a la 
posibilidad de elaborar una crítica epistemológica que, en lugar de "bastarse a sí misma", contribuya a la problematización ontológico-política respecto de la actualidad. Consideramos, entonces, que la problematización del saber económico, en su articulación con la emergencia de las formas de racionalización del ejercicio del gobierno, se configura por medio de lo que hemos denominado como crítica de las formas de objetivación.

A partir de la lectura propuesta, planteamos una serie de interrogantes que jalonan nuestro trabajo: ¿podemos criticar de modo radical la gubernamentalidad neoliberal si pasamos por alto las mutaciones que tuvieron lugar en el discurso de la economía política a lo largo del siglo XX? Justamente, en lo que respecta al neoliberalismo, ¿podemos problematizarlo sin realizar una crítica de las formas de objetivación inmanentes al discurso económico? "Capital humano", "gestión", "competencia", "eficacia y eficiencia", son algunos de los objetos y conceptos cuya constitución debe ser remitida al discurso de la economía neoliberal, así como la modalidad enunciativa sobre la que hunde sus raíces la figura del "experto". De no afrontar esta tarea, nos exponemos al peligro de quedar entrampados -al desplegar nuestra críticaen el tipo de racionalidad política que pretendemos cuestionar.

\section{Referencias}

Benente, M. (2014). La concepción del derecho en el análisis del biopoder. Tesis doctoral. Universidad de Buenos Aires. (Sin publicar).

Botticelli, S. (2014). A todos y a cada uno. La gubernamentalidad neoliberal en la administración estatal. Tesis doctoral. Universidad de Buenos Aires. (Sin publicar).

Cadahia, L., y Blengino, L. (2018). Pueblo y heterogeneidad: claves para una gubernamentalidad popular. J.L., Villacañas y R. Castro (Eds.), Foucault y la historia de la filosofía (pp. 419-439). Madrid: Dado Ediciones.

Castro, E. (1995). Pensar a Foucault: interrogantes filosóficos de La arqueología del saber. Buenos Aires: Editorial Biblos.

Castro, E. (2011). Lecturas foucaultianas. Una historia conceptual de la biopolitica. Buenos Aires: Ediciones UNIPE. 
Castro-Gómez, S. (2010). Historia de la gubernamentalidad. Razón de Estado, liberalismo y neoliberalismo en Michel Foucault. Bogotá: Siglo del Hombre Editores.

D’Alessandro, D. (2011). Filosofie della biopolitca. Figura e problemi. Tesis doctoral. Università degli Studi di Salerno. (Sin publicar).

Fontana, A., y Bertani, M. (1997). Situation du Cours. F. Ewald, M. Bertani y A. Fontana (Eds.), M. Foucault, Il faut défendre la société: Cours au Collège de France 1975-1976 (pp. 247-263). Paris: Hautes Études (Gallimard-Seuil).

Foucault, M. (1966). Les mots et les choses. Une archéologie des sciences humaines. Paris: Éditions Gallimard.

Foucault, M. (1969). L’archéologie du savoir. Paris: Éditions Gallimard.

Foucault, M. (1976). Histoire de la sexualité 1. La volonté de savoir. Paris: Éditions Gallimard.

Foucault, M. (1990). Qu`est-ce que la Critique? (Critique et Aufklrärung). Bulletin de la Société française de Philosophie, 84(2), pp. 35-63.

Foucault, M. (1994a). Qu’est-ce que les Lumières?. Dits et écrits. 1954-1988. IV. 1980-1988 (pp. 562-578). Paris: Éditions Gallimard.

Foucault, M. (1994b). La philosophie structuraliste permet de diagnostiquer ce qu’est “aujourd hui". Dits et écrits. 1954-1988. IV. I. 1954-1969 (pp. 580-584). Paris: Éditions Gallimard.

Foucault, M. (1994c). Qu'est-ce qu'un philosophe ?. Dits et écrits. 1954-1988. IV. I. 1954-1969 (pp. 552-553). Paris: Éditions Gallimard.

Foucault, M. (1994d). Nietzsche, la généalogie, 1’histoire. Dits et écrits. 19541988. II. 1970-1975. (pp. 136-156). Paris: Éditions Gallimard.

Foucault, M. (1997). "Il faut défendre la société". Cours au Collège de France. 1976. Paris: Éditions Gallimard SEUIL.

Foucault, M. (2004a). Naissance de la biopolitique. Cours au Collège de France. 1978-1979. Paris: Éditions Gallimard SEUIL.

Foucault, M. (2004b). Sécurité, Territoire, Population. Cours au Collège de France. 1977-1978. Paris: Éditions Gallimard SEUIL. 
Foucault, M. (2008). Le gouvernement de soi et des autres. Cours au Collège de France. 1982-1983. Paris: Éditions Gallimard SEUIL.

Foucault, M. (2011). Leçons sur la volonté de savoir. Cours au Collège de France. 1970- 1971. Paris: Éditions Gallimard SEUIL.

Giordano, C. (2007). Un'idea di filosofia. Michel Foucault, dalla critica dell ’antropologia all'etica de la cura di sé. Tesis doctoral. Università degli Studi di Napoli Federico II. (Sin publicar).

Gros, F. (2008). Situation du Cours. Le gouvernement de soi et des autres. Cours au Collège de France. 1982-1983 (pp. 348 - 361). Paris: Éditions Gallimard SEUIL.

Gutting, G. (1989). Michel Foucault's archaeology of scientific reason. Cambridge: Cambridge University Press.

Lazzarato, M. (2005). Biopolitique et bioéconomie. Multitudes, 22, pp. 1-9.

Mascaretti, G. M. (2014). Michel Foucault on Problematization, Parrhesia and Critique. Materiali Foucaultiani, 3(5-6), pp. 135-154.

Mauer, M. (2015). Foucault et le problème de la vie. Paris: Publications de la Sorbonne.

Méndez, P. (2017). Wilhelm Röpke y la espiritualidad del neoliberalismo. Astrolabio, 18, pp. 112-146.

Muhle, M. (2012). Histoire(s) de la vie de Canguilhem à Foucault. A.A.V.V., Epistemology and History. From Bachelard and Canguilhem to Today's History of Science (pp. 187-196). Berlin: Max Planck Institut for the History of Science.

Nosetto, L. (2010). Discursos y estrategias. Michel Foucault y la política. Tesis doctoral. Universidad de Buenos Aires. (Sin publicar).

Oksala, J. (2013). Neoliberalism and Biopolitical Governmentality. J. Nilsson y S-O. Wallenstein (Eds.), Foucault, Biopolitics and Governmentality (pp. 53-72). Huddinge: Södertörn University.

Raffin, M. (2014). Natureza e política: uma tensão no pensamento de Michel Foucault. Ágora Filosófica, 1(2), pp. 116-143. 
Raffin, M. (2015). La verdad y las formas políticas: la lectura temprana de la tragedia de Edipo en Michel Foucault. Anacronismo e irrupción: Revista de teoría y filosofía política clásica y moderna, 5(8), pp. 54-78.

Sabot, P. (2016). De Foucault à Macherey, penser les normes. Methodos. Savoirs et textes, 16. [En ligne], 16 | 2016, mis en ligne le 29 février 2016. URL : http://methodos.revues.org/4652 ; DOI : 10.4000/methodos.4652

Sacchi, E. (2017). Neoliberalismo, gubernamentalidad y mnemotécnicas de la crueldad. El arco y la lira. Tensiones y Debates, 5, pp. 47-63.

Sauquillo, J. (2004). La radicalización del uso público de la razón. (Foucault, lector de Kant). Daimon. Revista internacional de filosofía, 33, pp. 167-185.

Villacañas, J.L., Castro, R. (2018). Prólogo: fragmentos filosóficos, talleres históricos. J.L. Villacañas y R. Castro. (Eds.), Foucault y la historia de la filosofía (pp. 5-27). Madrid: Dado Ediciones.

Wallenstein, S-O. (2013). Introduction: Foucault, Biopolitics, and Governmentality. Nilsson y S-O. Wallenstein (Eds.), Foucault, Biopolitics and Governmentality (pp. 7-34). Huddinge: Södertörn University. 